\title{
Role of MexZ and PA5471 in transcriptional regulation of mexXY in Pseudomonas aeruginosa
}

Correspondence
Yoshiaki Ishigatsubo
ishigats@med.yokohama-cu.ac.jp

Received 10 March 2009

Revised 29 June 2009

Accepted 3 July 2009

\author{
Masaki Yamamoto, ${ }^{1}$ Atsuhisa Ueda, ${ }^{1}$ Makoto Kudo, ${ }^{1}$ Yasuhiro Matsuo, \\ Jun Fukushima, ${ }^{2}$ Taiji Nakae, ${ }^{3}$ Takeshi Kaneko ${ }^{4}$ and Yoshiaki Ishigatsubo ${ }^{1}$ \\ ${ }^{1}$ Department of Internal Medicine and Clinical Immunology, Yokohama City University Graduate \\ School of Medicine, Yokohama 236-0004, Japan
}

\begin{abstract}
MexXY, a drug efflux pump in Pseudomonas aeruginosa, confers resistance to aminoglycoside antibiotics. We recently reported that MexZ binds to the promoter region of the mexXY operon. Electrophoretic mobility shift assay (EMSA) using recombinant MexZ and oligonucleotide probes prepared from the intergenic region between mex $Z$ and mex $X$ revealed that MexZ binds to a 20 bp palindromic sequence. Culture of $P$. aeruginosa in the presence of tetracycline induced higher levels of MexX and MexZ, as measured by immunoblotting and EMSA, than in the absence of antibiotics. When MexZ was expressed by a mex $Z$ expression plasmid, the plasmid-borne MexZ repressed drug-induced MexX production, further confirming that MexZ acts as a repressor of the mexXY operon. PA5471 protein has been reported to be essential for drug-induced MexXY production. Similarly to that report, we observed that plasmid-borne PA5471 induced both MexX and MexZ production in PAO1 cells. Interestingly, interaction between MexZ and PA5471 was observed in a yeast two-hybrid assay. Furthermore, EMSA and in vitro transcription assays revealed that interaction between PA5471 and MexZ reduced MexZ DNA-binding ability, leading to $m e x X Y$ transcription. These findings contribute to the understanding of the molecular mechanisms underlying the transcriptional regulation of mex $Z$ and mexXY by drug-induced PA5471 expression.
\end{abstract}

\section{INTRODUCTION}

Pseudomonas aeruginosa is a major opportunistic pathogen in patients with chronic respiratory disease or in immunocompromised hosts. This bacterium shows intrinsic and acquired resistance to structurally and functionally diverse antimicrobial agents. Among several drug-resistance mechanisms of bacteria (McDermott et al., 2003; Poole, 2002b; Wright, 2003), drug efflux pumps are notable in that the transporters export a wide range of xenobiotics, including antimicrobial agents (Poole, 2005a). Among the efflux-pump family, the resistance-nodulation cell-division family transporters are unique to Gramnegative bacteria, and they contribute to the efflux of, and resistance to, antibiotics (Poole, 2002a). To date, seven different resistance-nodulation cell-division efflux pumps have been recognized (Mima et al., 2005; Poole, 2005a),

Abbreviations: EMSA, electrophoretic mobility shift assay; HRP, horseradish peroxidase. and among them, MexAB-OprM and MexXY-OprM play major roles in the natural antimicrobial resistance of $P$. aeruginosa (Poole, 2001). Although these pumps share common substrates (Masuda et al., 2000a; Okamoto et al., 2002), production of MexXY contributes largely to aminoglycoside resistance in $P$. aeruginosa (Hocquet et al., 2006; Masuda et al., 2000a, b; Okamoto et al., 2002; Poole, 2005b). Furthermore, their expression profiles are different: MexAB-OprM is constitutively produced in wild-type $P$. aeruginosa and overexpressed on mutation of the regulatory gene, whereas MexXY is produced in response to antimicrobial agents that target the ribosome (Jeannot et al., 2005; Masuda et al., 2000b).

We previously reported that MexZ binds to the mexZmexX intergenic DNA that contains the putative mexXY promoter (Matsuo et al., 2004). Because of its structural homology to other efflux-pump repressors, such as AmrR in Burkholderia pseudomallei and MtrR in Neisseria gonorrhoeae (Hagman et al., 1995; Klein et al., 1991; Ma 
et al., 1996), MexZ is assumed to be a repressor of mexXY transcription, and the following results support this assumption (Llanes et al., 2004; Morita et al., 2006; Vogne et al., 2004; Westbrock-Wadman et al., 1999): (i) experimentally derived and clinical isolates of aminoglycoside-resistant $P$. aeruginosa had a mutation in mexZ or mexZ-mexX intergenic DNA (Llanes et al., 2004; Vogne et al., 2004); and (ii) expression of plasmid-borne MexZ reduced the expression of mexY mRNA (Hocquet et al., 2006).

Morita et al. (2006) studied the role of the PA5471 gene in MexXY expression (Morita et al., 2006) and found that antibiotic-mediated MexXY induction was compromised in strains with an impaired PA5471 gene (Morita et al., 2006). Like MexXY expression, PA5471 expression is drug inducible, and its presence seems to be necessary for druginduced MexXY expression. Therefore, PA5471 somehow contributes to the transcription of mexXY, probably by modulating the function of MexZ. However, it is not known how PA5471 interacts with MexZ to promote the transcription of mexXY.

To elucidate the molecular mechanisms of MexXY induction in $P$. aeruginosa, we studied the expression of MexX and MexZ, the binding of MexZ to the mexX promoter, and the interaction between MexZ and PA5471 in a laboratory strain and a clinically isolated $P$. aeruginosa strain.

\section{METHODS}

Bacterial strains and growth conditions. The bacterial strains and plasmids used are listed in Table 1. Bacterial cells were cultured in Luria broth [LB; $10 \mathrm{~g}$ tryptone (Becton Dickinson), $5 \mathrm{~g}$ yeast extract (Becton Dickinson) and $10 \mathrm{~g} \mathrm{NaCl}$ per litre $\mathrm{H}_{2} \mathrm{O}$ ] and on LB agar [LB containing $1.5 \%(\mathrm{w} / \mathrm{v})$ agar] at $37{ }^{\circ} \mathrm{C}$. The medium was supplemented with antibiotics as needed. Clinically isolated strain \#21 of $P$. aeruginosa was employed to assess the mobility of MexZ-DNA complexes. The pEX1.8 plasmid and its derivatives were maintained in Escherichia coli and $P$. aeruginosa PAO1 cells in medium supplemented with $100 \mu \mathrm{g}$ ampicillin (Sigma) $\mathrm{ml}^{-1}$ and $200 \mu \mathrm{g}$ carbenicillin (CAR) (Wako) $\mathrm{ml}^{-1}$, respectively.

Preparation and manipulation of DNA. Standard protocols described by Ausubel et al. (1995) were used for restriction endonuclease digestion, ligation, transformation, plasmid isolation and agarose gel electrophoresis (Ausubel et al., 1995). Plasmid DNAs were prepared from E. coli by using the Nucleospin Plasmid kit (Macherey-Nagel) or the Plasmid Midi kit (Qiagen) according to the protocols provided by the manufacturers. The genomic DNA of $P$. aeruginosa was extracted by following the protocol of Barcak et al. (1991). The endonuclease-digested DNA fragments were purified

Table 1. Bacterial strains and plasmids used

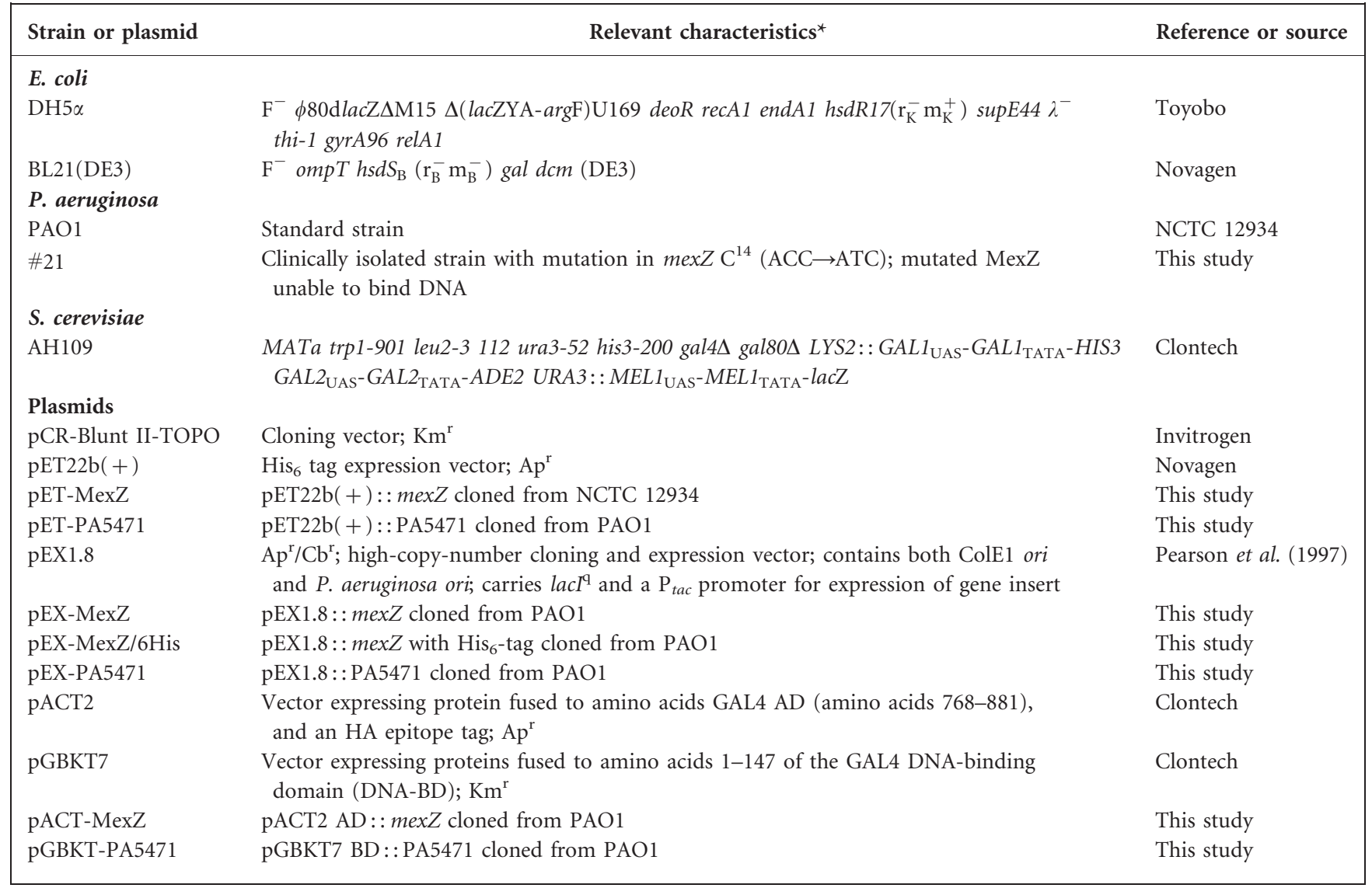

${ }^{\star} \mathrm{Ap}^{\mathrm{r}}$, ampicillin resistant; $\mathrm{Km}^{\mathrm{r}}$, kanamycin-resistant; $\mathrm{Cb}^{\mathrm{r}}$, carbenicillin resistant. 
with a Sephaglas BandPrep kit (GE Healthcare). PCR products were purified with a StrataPrep PCR Purification kit (Stratagene). Competent $P$. aeruginosa cells were prepared as described by Smith \& Iglewski (1989). Competent E. coli BL21(DE3) cells and Competent High DH5 $\alpha$ cells were purchased from Novagen and Toyobo, respectively.

Plasmid construction and transformation. All primers used for cloning of mexZ or PA5471 are shown in Table 2. To construct pCRMexZ(EP) plasmids, mexZ and PA5471 were amplified by PCR with the primer set E-mexZ-s/P-mexZ-as; for plasmids pCR-MexZ(NS), pCR-PA5471(EP), pCR-PA5471(NX), and pCR-MexZ(ES) similar amplifications were performed with $\mathrm{N}$-mexZ-s/S-mexZ-as, EPA5471-s/P-PA5471-as, N-PA5471-s/X-PA5471-as, and E-mexZ-s/SmexZ-as, respectively, by a standard PCR method (Ausubel et al., 1995). The PCR products were cloned into plasmid pCR-BluntIITOPO (Invitrogen) for nucleotide sequencing and further plasmid construction. To construct plasmid pET-MexZ, pCR-MexZ(NS) was digested with NdeI and SalI. The resulting DNA fragment was purified from agarose gel and cloned into the $\mathrm{Ndel} / \mathrm{Xhol}$ site of pET22b $(+)$. To construct pEX-MexZ, pCR-MexZ(EP) was digested with EcoRI and PstI. The resulting DNA fragment was gel-purified and cloned into the EcoRI/PstI site of pEX1.8. To construct pEXMexZ/6His, His-tagged mex $Z$ was amplified by PCR with the primer set E-mexZ-s/P-mexZ-his-as and pET-MexZ as the template. To construct pEX-PA5471 and pGBKT-PA5471, pCR-PA5471(EP) was digested with EcoRI and PstI. The resulting DNA fragment was gelpurified and cloned into the EcoRI/PstI site of pEX1.8 and pGBKT7. To construct pET-PA5471, pCR-PA5471(NX) was digested with NdeI and XhoI. The resulting DNA fragment was gel-purified and cloned into the NdeI/XhoI site of pET22b(+). To construct pACT2-MexZ, pCR-MexZ(ES) was digested with EcoRI and SalI. The resulting DNA fragment was gel-purified and inserted into the EcoRI/XhoI site of pACT2. $P$. aeruginosa was transformed with the plasmids by electroporation as described by Smith \& Iglewski (1989) using a Gene Pulser II and a Pulse Controller II (Bio-Rad). Saccharomyces cerevisiae strain $\mathrm{AH} 109$ cells, used for yeast two-hybrid assay, were transformed by the lithium acetate method, as described in the manufacturer's protocol.
Recombinant proteins. E. coli BL21(DE3) cells harbouring recombinant MexZ (reMexZ) or recombinant PA5471 (rePA5471) were grown at $37^{\circ} \mathrm{C}$ while being rotated at 160 r.p.m.; $1 \mathrm{mM}$ IPTG was added when the $\mathrm{OD}_{600}$ reached 0.6 , and the cells were incubated for an additional $3 \mathrm{~h}$. The bacterial cells were disintegrated by sonic oscillation in buffer A [50 mM sodium phosphate ( $\mathrm{pH} 7.5), 300 \mathrm{mM}$ $\mathrm{NaCl}$ and $20 \%(\mathrm{v} / \mathrm{v})$ glycerol]. The cell lysate was centrifuged at $100000 \mathrm{~g}$ at $4{ }^{\circ} \mathrm{C}$ for $60 \mathrm{~min}$, and the clear supernatant was applied to a HisBind Resin column (Novagen). After being washed with buffer A three times, the column was eluted with buffer A containing $300 \mathrm{mM}$ imidazole, and the eluate was dialysed against buffer B [20 mM Tris/ $\mathrm{HCl}$ (pH 7.5), $0.2 \mathrm{mM}$ EDTA, $1 \mathrm{mM}$ DTT, $1 \mathrm{mM} \mathrm{MgCl}_{2}, 100 \mathrm{mM}$ $\mathrm{NaCl}$ and $20 \%(\mathrm{v} / \mathrm{v})$ glycerol].

\begin{abstract}
Antibodies. To raise polyclonal antibody against reMexZ, the protein preparation was mixed with Freund's complete adjuvant and injected into a rabbit. Serum samples were collected 80 days after injection, and the anti-reMexZ antibody was affinity-purified through CNBr-Sepharose 4B columns coupled with reMexZ. Anti-MexX antibody was prepared as previously reported (Matsuo et al., 2004). Horseradish peroxidase (HRP)-conjugated anti-rabbit IgG antibody was supplied by GE Healthcare. Preimmune rabbit IgG was obtained from Upstate Biotechnology.
\end{abstract}

\begin{abstract}
Detection of mutations in the mexZ gene and mexZ-mexX intergenic DNA. The clinically isolated clone \#21 was subjected to sequencing analysis to detect possible mutations in the mexZ gene and mexZ-mexX intergenic DNA. The DNA fragment corresponding to the mexZ-mexX region was PCR amplified with the primer set mexZ$\mathrm{X}$-s/mexZ-as with genomic DNA isolated from clone $\# 21$ as a template. The DNA fragments were cloned into pCR-BluntII-TOPO (Invitrogen) and subjected to sequence analysis.
\end{abstract}

Preparation of $\boldsymbol{P}$. aeruginosa cell extracts. P. aeruginosa cells were cultured in $2 \mathrm{ml} \mathrm{LB}$ for $16 \mathrm{~h}$ at $37^{\circ} \mathrm{C}$ and then centrifuged at $20000 \mathrm{~g}$ at $4{ }^{\circ} \mathrm{C}$ for $5 \mathrm{~min}$; the pellet was then suspended in $100 \mu \mathrm{l}$ lysis buffer [10 mM Tris/HCl (pH 7.5), $10 \mathrm{mM} \mathrm{KCl,} 0.2 \mathrm{mM}$ EDTA, $0.2 \mathrm{mM}$ EGTA and $0.5 \mathrm{mM}$ DTT]. Cells were disintegrated by sonic oscillation with 15 repeated bursts of $1 \mathrm{~min}$ each, total $15 \mathrm{~min}$, at

Table 2. Primer sequences used for DNA amplification

\begin{tabular}{|c|c|c|}
\hline Primer & Sequence $\left(5^{\prime} \rightarrow 3^{\prime}\right)^{\star}$ & Purpose \\
\hline E-mexZ-s & GAATTCATGGCCAGGAAAACCAAAG; EcoRI & PCR cloning of mexZ \\
\hline N-mexZ-s & 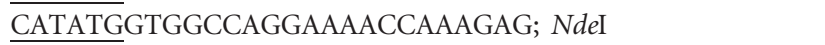 & PCR cloning of mexZ \\
\hline P-mexZ-as & CTGCAGTCAGGCGTCCGCCAGCAACA; PstI & PCR cloning of mexZ \\
\hline S-mexZ-as & GTCGACTAAGGCGTCCGCCAGCAACAGGTAGGGAG; SalI & PCR cloning of mexZ \\
\hline P-mexZ-his-as & 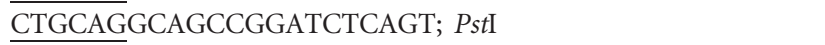 & PCR cloning of mex $Z / 6 \mathrm{His}$ \\
\hline E-PA5471-s & GAATTCATGGGCAACTACATCAAGCCTTTGTCCGC; EcoRI & PCR cloning of PA5471 \\
\hline N-PA5471-s & CATATGATGGGCAACTACATCAAGCCTTTGTCCGCG; NdeI & PCR cloning of PA5471 \\
\hline P-PA5471-as & CTGCAGTCATCGGCAGCACTCCCCAC; PstI & PCR cloning of PA5471 \\
\hline $\mathrm{X}-\mathrm{PA} 5471-\mathrm{as}$ & CTCGAGGCATCGGCAGCACTCCCCACGGGTCTTGTA; XhoI & PCR cloning of PA5471 \\
\hline U1 & CTTTGGTTTTCCTGGCCACTGAACGT & EMSA probe \\
\hline D1 & GTCCATTGGATGTGCATGGGTG & EMSA probe \\
\hline mexZ-X for Seq & GTCCATTGGATGTGCATGGGTG & Sequence analysis \\
\hline mexZ-as for Seq & CTGCAGTCAGGCGTCCGCCAGCAACA & Sequence analysis \\
\hline T7-185-MexX-s & CTAATACGACTCACTATAGGGAGAAACGCCAATTAATTAATC & In vitro transcription assay \\
\hline MexX-us & TCACTGGCCCGCCGGCGAGGC & In vitro transcription assay \\
\hline
\end{tabular}

${ }^{\star}$ Underlined sequences indicate restriction sites; the corresponding restriction endonuclease is indicated. The bold sequence indicates the T7 promoter region. 
power setting 10 with a Sonifier 450D (Branson Ultrasonics) with a cup horn in which cold water $\left(4{ }^{\circ} \mathrm{C}\right)$ was circulated. Intact cells were removed by centrifugation at $20000 \mathrm{~g}$ at $4{ }^{\circ} \mathrm{C}$ for $5 \mathrm{~min}$. The amount of protein was determined by the Bradford assay (Bio-Rad), and the final protein concentration of each sample was adjusted to $10 \mathrm{mg}$ $\mathrm{ml}^{-1}$ with lysis buffer for electrophoretic mobility shift assay (EMSA) and immunoblotting assays.

EMSA, competition assay and supershift assay. EMSA was carried out as described by Ausubel et al. (1995). Briefly, the probes were prepared by the annealing of four sets of complementary oligonucleotides (Fig. 1). Probe $(-183 /+17$ ) was prepared by a standard PCR method and gel purification. Binding of recombinant protein and cell extract was performed at $20{ }^{\circ} \mathrm{C}$ for $30 \mathrm{~min}$ in $10 \mu \mathrm{l}$ binding buffer $[10 \mathrm{mM}$ Tris/ $\mathrm{HCl}(\mathrm{pH} 8.0), 100 \mathrm{mM} \mathrm{NaCl}, 16 \mathrm{mM}$ $\mathrm{MgCl}_{2}, 0.5 \mathrm{mM}$ EDTA, $14 \%$ (v/v) glycerol and $1 \mathrm{mM}$ DTT] containing $500 \mathrm{fmol}$ of each probe end-labelled with $\left[\gamma_{-}{ }^{32} \mathrm{P}\right] \mathrm{ATP}$ (GE Healthcare), $1 \mu \mathrm{g}$ poly(dI-dC) (Amersham Biosciences) and $1 \mu \mathrm{g}$ BSA. For the competition assay, a 50-fold excess of an appropriate unlabelled probe was added to the binding reaction mixtures. For the supershift assay, the recombinant protein and cell extract were preincubated with a rabbit IgG or an anti-MexZ antibody for $20 \mathrm{~min}$ before being mixed with the probe. Gel electrophoresis was carried out at $120 \mathrm{~V}$ for $2 \mathrm{~h}$ at $4{ }^{\circ} \mathrm{C}$ in $0.5 \times$ TBE buffer $[45 \mathrm{mM}$ Tris $/ \mathrm{HCl}$ $(\mathrm{pH} 8.0), 45 \mathrm{mM}$ boric acid and $1 \mathrm{mM}$ EDTA] on a $5 \%(\mathrm{w} / \mathrm{v})$ polyacrylamide gel.

Immunoblotting assay. The extracts from $P$. aeruginosa cells were subjected to SDS-PAGE. The proteins were transferred onto Immobilon-P membranes (Millipore). After blocking non-specific binding sites with $5 \%(\mathrm{w} / \mathrm{v})$ skim milk in TBS buffer $[10 \mathrm{mM}$ Tris/
$\mathrm{HCl}(\mathrm{pH} \mathrm{7.5)}$ in $150 \mathrm{mM} \mathrm{NaCl}$ for $2 \mathrm{~h}$ at room temperature, the membrane was probed for $1 \mathrm{~h}$ with primary antibody diluted $1: 4000$ in TBS containing $0.1 \%(\mathrm{w} / \mathrm{v})$ skim milk. The membrane was next probed for $1 \mathrm{~h}$ with a $1: 10000$ dilution of the secondary HRPconjugated anti-rabbit IgG antibody. After the membrane had been washed three times with TBS containing $0.05 \%(\mathrm{v} / \mathrm{v})$ Tween 20 , the immunoreactive protein bands were visualized by using ECL Plus Western blotting Reagents (GE Healthcare) and an LAS-3000 mini luminescent image analyser (Fujifilm).

In vitro transcription assay. A 1396 bp DNA fragment containing a phage T7 promoter at its $5^{\prime}$ end, the $185 \mathrm{bp} 5^{\prime}$-flanking sequence of mexX (including the MexZ-binding site) and the mexX gene was amplified from $P$. aeruginosa PAO1 genomic DNA by PCR using KOD plus (Toyobo) and the primer set T7-185-MexX-s/MexX-us. The PCR product was gel-purified with a Sephaglas BandPrep kit (GE Healthcare), and quantified with a GeneQuantII spectrophotometer (Pharmacia Biotech). In vitro transcription from this template was conducted with a CUGA7 in vitro transcription kit (Nippon Genetech) according to the manufacturers' protocol, except that the amount of the template was reduced to $5 \mathrm{pg}$ (final concentration $15 \mathrm{nM}$ ), and half the suggested amount of CUGA7 enzyme solution was used. To assess the impact of the MexZ repressor on transcription from these templates, purified MexZ was added to the reaction mixtures. To assess the impact of PA5471 on MexZ repressor activity in this assay, purified PA5471 was also added to the reaction mixtures. A control reaction was also carried out with PA5471 alone. In all instances, reactions were allowed to proceed for $2 \mathrm{~h}$ at $37^{\circ} \mathrm{C}$. After DNase digestion for $30 \mathrm{~min}$, the RNA products were purified by using Isogen (Nippon Gene) and ethanol precipitation. Purified RNA products were resuspended in $20 \mu \mathrm{l}$ diethylpyrocarbonate-treated

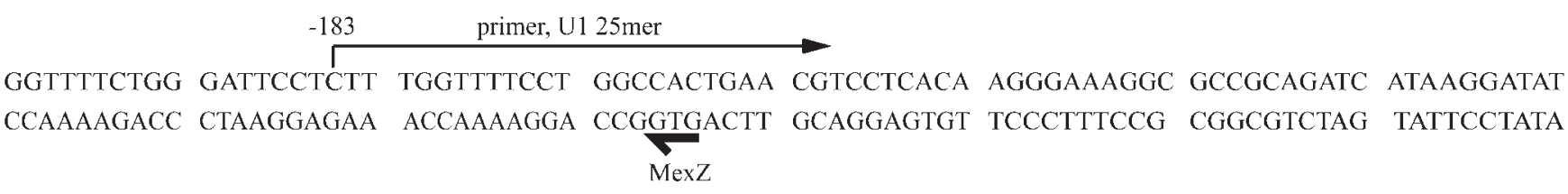

$-104$

$-66$

GTTACAGAAA CGCCAATTAA TTAATCACTC ATGATTGATT ATATGCTACT GTCG'GCCGC TCGTGAGTTG CGGTGCCCTT CAATGTCTTT GCGGTTiAatT A ATtAGTGAG TACTAACTAA TATACGATGA CAGCGCGGCG AGCACTCAAC GCCACGGGAA

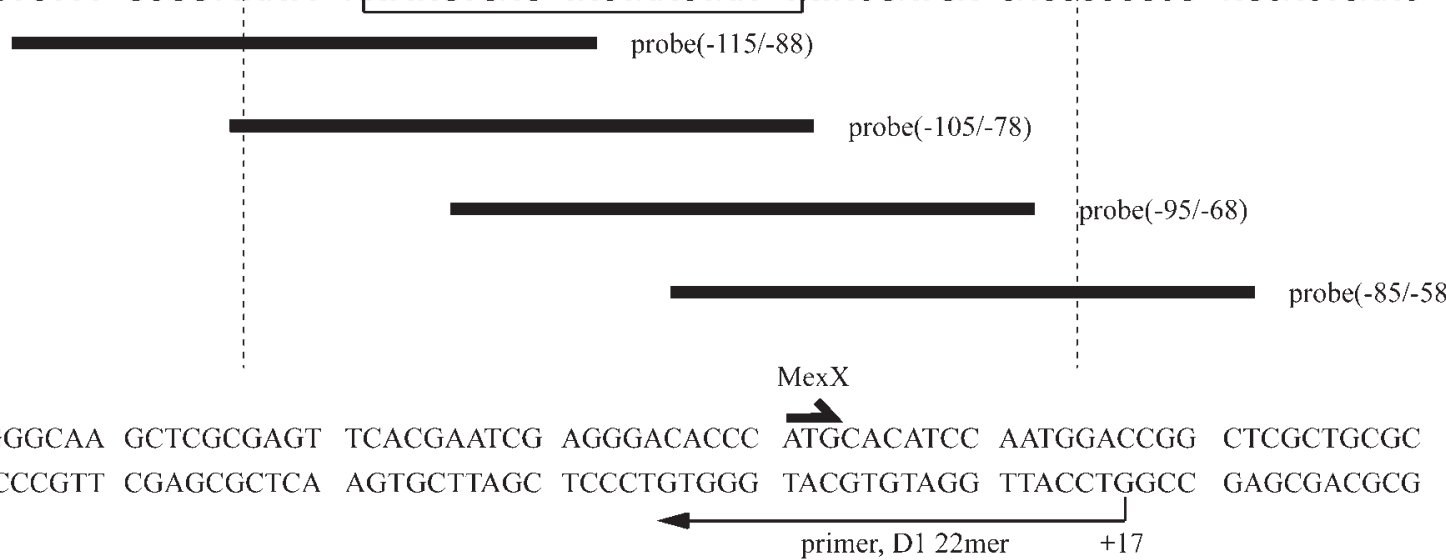

Fig. 1. MexZ binds to a $20 \mathrm{bp}$ palindromic sequence. The nucleotide sequence of the mexZ-mexXY intergenic region is shown. The translation initiation site of MexX is designated +1 . Thin arrows indicate the PCR primers (sense primer U1 above, anti-sense primer D1 below) used for the preparation of probe $(-183 /+17)$. Thick arrows indicate the translation start codons for MexZ and MexX. The sequences of the oligonucleotide probes for EMSA are bold underlined. The 20 bp palindromic sequence is boxed. 
water, mixed with the same volume of $8 \mathrm{M}$ urea sample buffer, heated to $55{ }^{\circ} \mathrm{C}$ for $5 \mathrm{~min}$, and then separated by electrophoresis in $4 \%$ $(\mathrm{w} / \mathrm{v})$ polyacrylamide gel in $1 \times \mathrm{TBE}$ buffer. Size-separated RNA products were visualized by ethidium bromide staining.

Yeast two-hybrid assay. A yeast two-hybrid assay was performed with Matchmaker GAL4 Two-Hybrid System 2 (Clontech Laboratories). We constructed full-length MexZ and PA5471 expression vectors, pACT-MexZ and pGBKT-PA5471. S. cerevisiae AH109 cells were transformed with derivatives of both pACT2 and pGBKT7 by the lithium acetate method, as described in the manufacturer's protocol. Transformants were inoculated on selective minimal plates [SD medium containing $2 \%(\mathrm{w} / \mathrm{v})$ agar and lacking leucine and tryptophan] and grown for 4 days at $30{ }^{\circ} \mathrm{C}$ to select clones transformed by both derivatives. Then, colonies were picked up and grown for 4 days at $30^{\circ} \mathrm{C}$ on selective maximal plates [SD medium containing $2 \%(\mathrm{w} / \mathrm{v})$ agar and lacking adenine, histidine, leucine and tryptophan] to select clones expressing the reporter genes $A D E$ and HIS3.

\section{RESULTS AND DISCUSSION}

\section{Refining of the Mexz-binding site}

We reported previously that MexZ binds to the sequence between -104 and $-66 \mathrm{bp}$ upstream of the MexXY translation initiation site (Matsuo et al., 2004). For further characterization of MexZ binding to the mexX promoter, EMSA was performed using reMexZ and four types of ${ }^{32} \mathrm{P}-$ labelled oligonucleotide probe. The nucleotide sequences of each probe are shown in Fig. 1. Although reMexZ bound to both probe $(-115 /-88)$ and probe $(-105 /-78)$ (Fig. 2a, lanes 1 and 2), a competition assay revealed that reMexZ bound specifically to probe $(-105 /-78$ ) (lane 6), whereas the binding to probe $(-115 /-88)$ seemed to be non-specific (lane 5). Therefore, we used probe $(-105 /-78)$ to examine the DNA-binding ability of MexZ in the following EMSA. Probe(-105/-78) coded for a $20 \mathrm{bp}$ palindromic sequence (Fig. 1). Using a higher dose of reMexZ elicited another, much slower-mobility, band (as seen in Fig. 2e, lanes 5, 7 and 8, arrowhead). Although excessive accumulation of radiolabelled probes in the adjacent lanes sometimes interfered with the bands and made them hard to recognize (as seen in Fig. 2b, lane 7), we used reMexZ in EMSA at a dose that showed only a single band.

\section{Promotion of MexZ production by tetracycline, and MexZ binding to mexXY promoter region}

MexXY is not produced in $P$. aeruginosa cells when the bacteria are grown in the absence of antibiotics. Tetracycline (TET) and other ribosome-targeting antibiotics promote MexXY production, even at subinhibitory concentrations (Jeannot et al., 2005). The following lines of evidence on the regulation of MexXY production have been presented to date. The mexZ gene, located upstream of mexXY, encodes a repressor protein that suppresses transcription of mexXY (Dean et al., 2003; Llanes et al., 2004; Morita et al., 2006; Vogne et al., 2004; WestbrockWadman et al., 1999). In fact, purified MexZ has been shown by gel-shift assay to bind with a DNA fragment from the mexZ-mexX intergenic promoter region (Matsuo et al., 2004). A computer-aided similarity search of MexZ has revealed that MexZ is a member of the TETR family of repressors (Aires et al., 1999; Westbrock-Wadman et al., 1999), which forms a homodimer for DNA binding (Aramaki et al., 1995; Grkovic et al., 2001; Hillen \& Berens, 1994). We therefore hypothesized that MexZ represses the transcription of mexXY, except in the presence of an appropriate inducer molecule such as TET. To verify this hypothesis, we performed EMSA using the lysate from $P$. aeruginosa PAO1 cells grown in the absence of TET. Low-mobility bands, which probably corresponded to protein-DNA-complex bands, were faintly detected (Fig. 2b, lane 2). However, when EMSA was performed using the lysate from PAO1 cells grown in the presence of TET, the low-mobility bands were more clearly seen than in EMSA using lysate from the cells grown without antibiotics (Fig. 2b, lane 3). These TET-inducible protein-DNA complexes showed slightly higher mobility than the reMexZ-DNA complex (Fig. 2b, lane 1). Since reMexZ has a $\mathrm{His}_{6}$ tag, an effect of the $\mathrm{His}_{6}$ tag on the mobility of reMexZ could not be ruled out. To examine the effect of the $\mathrm{His}_{6}$ tag on mobility, we conducted EMSA using lysates of the clinically isolated $P$. aeruginosa \#21 transfected with pEX-MexZ or pEX-MexZ/6His. Because this strain had a mutation in mexZ (Table 1), endogenous MexZ was detectable by immunoblotting assay when cells were grown without antibiotics (Fig. 3, lane 5) but was unable to bind DNA (Fig. 2b, lane 4), even when cells were cultured with TET (data not shown). The locations of the shifted bands seen in the lanes of reMexZ and pEX-MexZ/ $6 \mathrm{His}$, and of the TET-inducible protein band and pEXMexZ, were identical (Fig. 2b, lanes 1 and 6, and lanes 3 and 5 , respectively). Then, to compare the precise binding sequences of the TET-inducible protein and reMexZ, EMSA was performed using six different mutant oligonucleotide probes as cold competitors (Fig. 2c, upper panel). The mutated probes $\mathrm{m} 2, \mathrm{~m} 4$ and $\mathrm{m} 6$ had only small effects on reMexZ binding to probe(-105/-78) DNA (Fig. 2c, lower panel), indicating that the nucleotide sequences present in these mutated bases were not important for reMexZ binding. A similar result was observed in a competition assay using the lysate from $\mathrm{PAO} 1$ cells grown with TET (data not shown). These results were consistent with the result that all the probes, except probe $(-105 /$ -78 ), failed to bind to reMexZ because they lacked the nucleotide sequences important for DNA binding (Figs 1 and $2 \mathrm{a}$ ).

In the next experiment, the presence or absence of MexZ in the PAO1 cell lysates was assayed by immunoblotting with anti-MexZ antibody. MexZ was undetectable in PAO1 cells grown without TET (Fig. 2d, Fig. 3, lane 1). TET-induced production of MexZ paralleled the production of MexX and increased in a time-dependent manner (Fig. 2d). We then performed a supershift assay using this antibody. The protein-DNA-complex bands seen in EMSA using reMexZ 
(a)

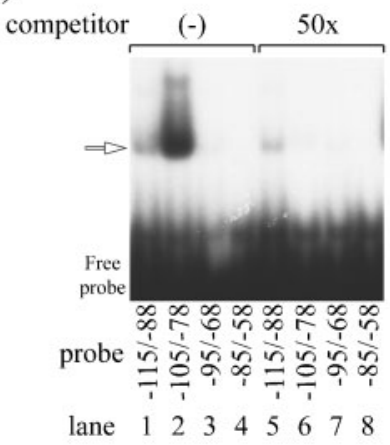

(b)



(c)
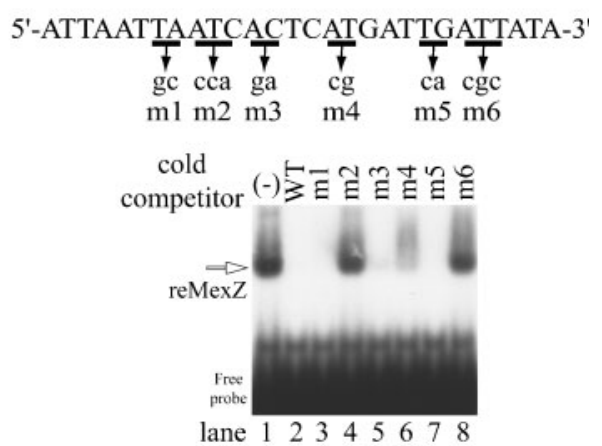

(d)

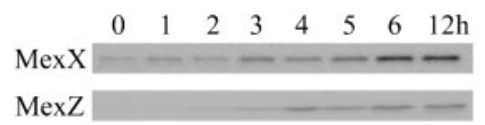

(e)

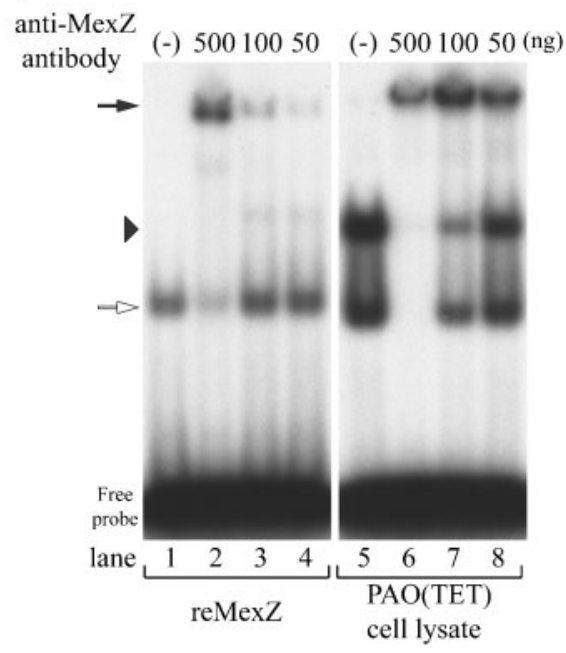

Fig. 2. Characterization of MexZ binding to the mexX promoter region. (a) EMSA indicates that recombinant MexZ (reMexZ) binds to probe(-115/-88) (lane 1) and probe(-105/-78) (lane 2), but not to probe(-95/-68) (lane 3) or probe(-85/-58) (lane 4). The competition assay indicated that reMexZ bound to probe(-105/-78) with the highest affinity (lane 6). (b) EMSA indicates reMexZ binding to probe $(-105 /-78$ ) (lanes 1 and 7, white arrow). When $P$. aeruginosa PAO1 was cultured in Luria broth without antibiotic agents, a shifted band was faintly detected by EMSA using probe(-105/-78) (lane 2, solid arrow). This shifted band was detected more clearly upon culture with tetracycline (TET) lysate (lane 3, solid arrow). To examine the effect of the $\mathrm{His}_{6}$ tag on mobility, EMSA was conducted using lysates of the clinically isolated $P$. aeruginosa \#21 and its pEX-MexZ or pEX-MexZ/6His transformants. Endogenous MexZ of \#21 could not bind to probe(-105/-78) (lane 4). The locations of the shifted bands seen in the lanes of pEX-MexZ (lane 5, solid arrow) and pEX-MEXz/6His (lane 6, white arrow) were identical to those of the TET-induced protein band (lane 3 ) and reMexZ (lane 1), respectively. (c) The sense oligonucleotide sequence of probe $(-105 /-78)$ is shown (upper panel). The arrows indicate bp substitutions in mutated oligonucleotide probes ( $\mathrm{m} 1, \mathrm{~m} 2, \mathrm{~m} 3$, $\mathrm{m} 4, \mathrm{~m} 5$ and $\mathrm{m} 6$ ). A competition assay was performed by using reMexZ and the mutated probes as cold competitors (lower panel). The same result wasobtained with an extract from TET-stimulated PAO1 cells. (d) MexX and MexZ expression, examined by immunoblotting using antibodies against MexX and MexZ. At the indicated times after incubation in LB medium containing TET $\left(2 \mu \mathrm{g} \mathrm{ml}^{-1}\right)$, extracts from PAO1 cells were subjected to immunoblotting. (e) By addition of an anti-MexZ antibody, both reMexZ-DNA complexes and TET-induced protein-DNA complexes (lanes 1-4 and 5-8, respectively, white arrow) were dosedependently supershifted (lanes 2, 3, 4, 6, 7 and 8, solid arrow). As a negative control, preimmune rabbit lgG was employed in the assay (data not shown). All experiments were performed two or three times, with similar results. 


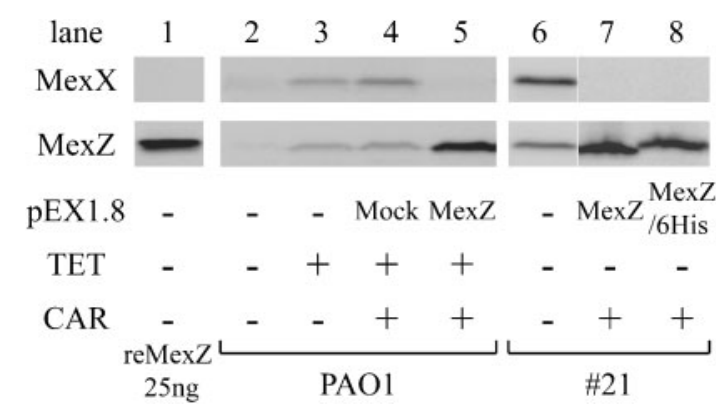

Fig. 3. Effect of tetracycline and recombinant MexZ on MexX expression: immunoblotting analysis of MexX and MexZ expression in $P$. aeruginosa PAO1 cells, clinically isolated strain \#21, and their transformants. As a control for the expression level of MexZ, recombinant MexZ (25 ng) was employed. MexX and MexZ were not expressed in PAO1 cells cultured in Luria broth without antibiotic agents (lane 2). TET (2 $\left.\mu \mathrm{g} \mathrm{ml}^{-1}\right)$ induced both MexX and MexZ expression (lane 3). Plasmid-borne MexZ repressed the TET-induced MexX expression (lane 5). Carbenicillin (CAR, $200 \mu \mathrm{g} \mathrm{ml}^{-1}$ ) and transformation with a pEX1.8 mock plasmid (lane 4) had no detectable effect on MexX and MexZ expression in $\mathrm{TET}^{+}$PAO1 cells. MexX and MexZ were expressed in clinically isolated $P$. aeruginosa, \#21 (lane 6), when the bacteria were grown in the absence of antibiotics. Endogenous MexZ of \#21 was not capable of DNA binding. Plasmid-borne MexZ and hisitidine-tagged MexZ cloned from PAO1 repressed the expression of MexX in \#21 (lanes 7 and 8, respectively).

and the lysate from TET-stimulated PAO1 cells were dosedependently supershifted by the addition of anti-MexZ antibody (Fig. 2e, lanes 2, 3, 4, 6, 7 and 8). The supershifted band in Fig. 2e, lane 6, is slightly fainter than that in lane 7. This is because some DNA-protein complexes were trapped in the upper parts of the well owing to the high protein concentration. A control experiment using preimmune rabbit IgG showed no detectable supershift (data not shown). Another, much slower-mobility band is seen in lanes 3, 4, 5, 7 and 8 . This slower-mobility band was often seen when the binding reaction was conducted in a more concentrated solution (see Fig. 4d, lane 2). We did not pursue the question of whether this slower-mobility band had a functional meaning.

Piecing together these results showed that the TET-induced protein was identical to MexZ. We concluded that TET stimulates MexZ production in $P$. aeruginosa PAO1, as reflected by the increased binding in EMSA and by the results of the immunoblotting analysis. Because MexZ is a member of the TetR family and it has been reported that one of the TetR family proteins, TtgR of Pseudomonas putida, has the ability to bind to several structurally unrelated molecules (Teran et al., 2006), we checked the possibility of direct interaction between MexZ and antibiotics, especially TET, by EMSA. However, addition of TET to the binding reaction solutions could not alter the shifted band of the reMexZ-DNA complex (data not shown).

\section{MexZ-mediated repression of mexXY transcription}

It has been reported that TET stimulates MexXY production (Jeannot et al., 2005) and induces the transcription of mexZ (Dean et al., 2003; Morita et al., 2006) in PAO1 cells. Our results also revealed that TET stimulated the production of MexZ, which binds with mexXY promoter DNA (Fig. 2b, Fig. 3, lane 3). Several reports have shown that some repressors of bacterial efflux pumps are auto-regulated (Sanchez et al., 2002a, b; Teran et al., 2006) and we thought this might be the case here. To clarify the role of MexZ again, we transformed PAO1 cells with the mexZ expression plasmid pEX-MexZ and examined the effects of plasmid-borne overproduced MexZ on MexX production; we observed that the plasmid-borne MexZ repressed MexX production in TET-stimulated PAO1 cells (Fig. 3, lane 5). A mock plasmid and CAR, which was used to select plasmids containing PAO1 cells, exerted no detectable effect on MexX and MexZ production (Fig. 3, lane 4). Thus, the role of MexZ as a repressor was firmly verified. Although TET stimulates both MexX and MexZ expression in PAO1 cells, TET-induced MexX expression may be regulated by a transcription mechanism that remains to be characterized.

\section{Effect of PA5471 on MexX expression and interaction with MexZ}

Recently, Morita et al. (2006) reported that ribosometargeting antibiotics induce the production of cytoplasmic PA5471 protein, which seems to be indispensable for the production of MexXY proteins (Morita et al., 2006). However, the precise function of PA5471 in the regulation of mexXY remains to be elucidated.

When PAO1 cells were transformed with the PA5471 expression plasmid pEX-PA5471, the plasmid-borne PA5471 promoted the production of both MexZ and MexX (Fig. 4a, lane 3), confirming the results of Morita et al. (2006). We performed an in vitro transcription assay using reMexZ, rePA5471 and a DNA fragment that contains a phage $\mathrm{T} 7$ promoter at its $5^{\prime}$ end, the mexZmexX intergenic sequence (including the MexZ-binding site) and the mex $X$ gene. mex $X$ transcription from the DNA fragment was considerably suppressed by reMexZ (Fig. 4b, lane 2) and slightly suppressed by rePA5471 (lane 5). The slight suppression due to rePA5471 (lane 5) might have been caused by the presence of residual uneluted cell extract components, such as RNases, because the rePA5471 was affinity-purified by resin. Suppression by reMexZ was reduced by the addition of rePA5471 (lanes 3 and 4), indicating that the PA5471-induced transcription was MexZ-dependent.

To further examine the possibility of interaction between PA5471 and MexZ, we performed a yeast two-hybrid assay of AH109 yeast cells. We constructed full-length MexZ and PA5471 expression vectors, pACT-MexZ and pGBK- 
(a)

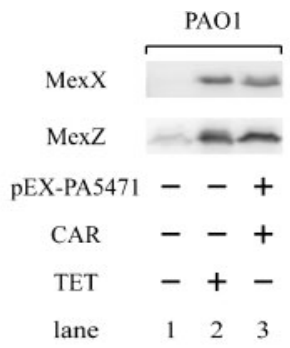

(c)

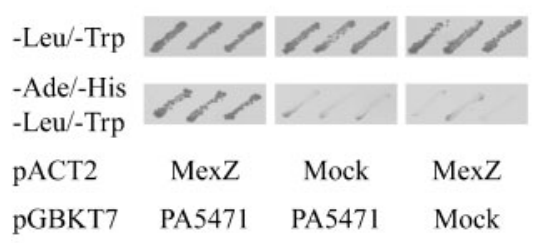

(e)

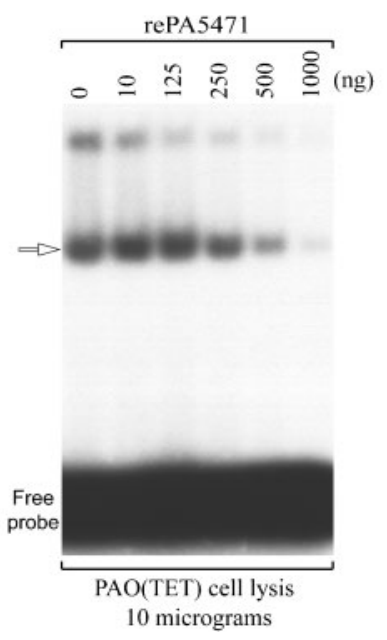

(b)

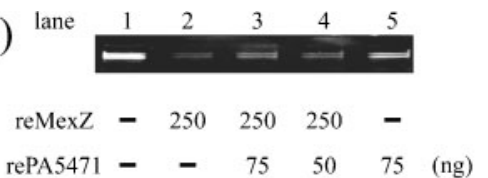

(d)
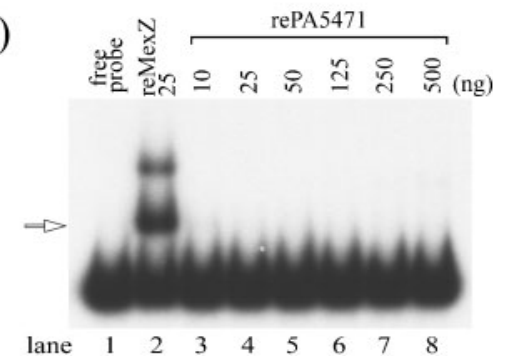

Fig. 4. $P A 5471$ binds to $M e x Z$ and relieves mexX repression. (a) PAOI cells were transformed with the PA5471 expression plasmid pEX-PA5471. Plasmid-borne PA5471 stimulated both MexX and MexZ expression (lane 3), as did TET (lane 2). (b) To elucidate whether PA5471 functionally inactivates MexZ, an in vitro transcription assay was performed. reMexZ inhibited transcription of the DNA fragment containing the MexZ-binding site (lane 2). This repression was dose-dependently reduced by the addition of recombinant PA5471 (rePA5471) (lanes 3 and 4). (c) To characterize the possible association between MexZ and PA5471, a yeast two-hybrid assay was performed. As a negative control, pACTmock and pGBKT-mock were used. Transformants containing both pACT-MexZ and pGBKT-PA5471 grew on maximal selective plates [SD medium containing $2 \%(\mathrm{w} / \mathrm{v})$ agar and lacking adenine, histidine, leucine, and tryptophan], indicating interaction between MexZ and PA5471. (d) To examine the possibility that PA5471 binds to the mexX promoter region, EMSA was performed with probe $(-183 /+17)$. reMexZ bound to the mex $X$ promoter region (bp -183 to +17 ) (lane 2, white arrow), but rePA5471 did not (lanes 38). (e) Recombinant PA5471 inhibited TETinduced MexZ binding to probe(-105/-78) (white arrow). Similar results were obtained using reMexZ.

PA5471, and then transformed the AH109 yeast cells with them. Yeast cells transformed with pACT and pGBK derivatives were selected using plates lacking leucine and tryptophan (Fig. 4c, upper panel). Because the interaction between MexZ and PA5471 could reconstitute GAL4 function, leading to the transcription of reporter genes $A D E$ and HIS3, only cells transformed with pACT-MexZ and pGBK-PA5471 could grow on the plates lacking adenine, histidine, leucine and tryptophan (Fig. 4c, lower panel). Thus, the interaction between MexZ and PA5471 was confirmed.

We also performed EMSA using probe $(-183 /+17)$, which has a $203 \mathrm{bp} 5^{\prime}$-flanking sequence of the mexX gene (Fig. 1), and recombinant proteins. Although reMexZ was capable of binding the probe(Fig. 4d, lane 2), rePA5471 failed to show binding (lanes 3-8). Because the shifted band of MexZ could not be observed in EMSAs performed with lysate from PA5471 overexpressing mutant (data not shown), in which the production of MexZ was increased, we examined the interaction between probe $(-105 /-78)$, PA5471 and MexZ by EMSA using extracts from TETstimulated PAO1 cells. Interestingly, rePA5471 inhibited the binding of MexZ to probe $(-105 /-78)$ in a dosedependent manner (Fig. 4e). Similar results could be obtained using reMexZ. Because immunoblotting analysis showed that the amount of MexZ in the cell lysate was far lower than in the amount of reMexZ used in our experiments (Fig. 3, lanes 1 and 3), it seemed that much more PA5471 was required to reduce the binding of MexZ in the cell lysate than in the recombinant form. However, because these two experiments were conducted under different conditions (with respect to protein concentration and composition of reaction mix) and the influence of the His $_{6}$-tag in reMexZ on the interaction with PA5471 could not be excluded, we thought it was inappropriate to compare these EMSA results. All these results revealed that PA5471 interacts with MexZ, blocks its function, and facilitates transcription of the mexXY operon. 
Transcriptional regulation of mexXY by the interaction of these proteins can be explained by comparison with the negative regulation of TetR-tetA, which is commonly used as a tool for selective target-gene regulation (Forster et al., 1999; Freundlieb et al., 1997; Gossen et al., 1995). In short, expression of TetA is under the tight control of TetR (Hillen \& Berens, 1994; Schnappinger \& Hillen, 1996) because high-level production of TetA is lethal for bacterial cells (Eckert \& Beck, 1989). Reduction of the affinity of TetR for tet $O$, which is accomplished by TetR and a tetracycline-magnesium complex, leads to the transcription of both tetA and tetR (Bertrand et al., 1983). Similarly, without antibiotics, production of MexXY is tightly controlled by MexZ. Although production of MexZ was not detected by immunoblotting, binding of MexZ to the mexZ-mexX intergenic promoter region could be seen in our EMSA even when cells were grown without antibiotics. MexZ loses its DNA-binding ability by forming a complex with drug-induced PA5471. This leads to the transcription of mexXY and mexZ, which was proved by the TETinduced parallel production of MexZ with MexX in immunoblotting (Fig. 2d). The production level of MexZ then becomes high enough to weaken the effect of PA5471, and transcriptional regulation of mexXY and mexZ by MexZ occurs again. By this regulatory system, the production levels of MexXY and MexZ can be maintained at a certain level as long as PA5471 is present. In fact, production of MexX and MexZ did not change at or after $12 \mathrm{~h}$ culture with TET in our immunoblotting analysis (data not shown). Once the PA5471-inducer molecule, such as TET, disappears, downregulation of mexX and mexZ promptly occurs. Using immunoblotting, we could observe parallel reduction of TET-induced MexX and MexZ production in PAO1 cells after removal of the TET from the culture medium (data not shown). This regulation mechanism is quite similar to the regulation of MexAB-OprM transcription by MexR, which interacts with PA3719, leading to loss of its DNA-binding ability (Daigle et al., 2007). However, the existence of shifted bands in our EMSAs when we used a lysate of PAO1 cells cultured with TET may seem contradictory if MexXY production is promoted by reducing the amount of MexZ bound to the promoter region. When the regulatory system described above is active, the production level of MexZ in each cell at each time point should fluctuate slightly higher or lower than equal to negate the effect of PA5471 to maintain the production level of MexX. The increase in binding of MexZ seen in the EMSA using PAO1 cultured with TET could be explained by slightly excess amounts of MexZ in some cells. In fact, when we conducted EMSA with lysates of PAO1 cells exposed to TET for $1-6 \mathrm{~h}$ at hourly intervals, the shifted bands of MexZ gradually became apparent with time (data not shown). There may be an alternative explanation for the existence of shifted bands in EMSAs using a lysate of cells cultured with TET. MexZ may be able to bind to antibiotics, such as TET, and the MexZ-antibiotic complex may be inactive as for other members of the TetR family. Because TET was not added to the EMSA binding reaction, TET in the cell lysate was diluted and might not have been present at a high enough concentration to influence the binding ability of MexZ. However, as previously described in this report, addition of TET to the binding reaction solutions could not alter the shifted band of reMexZ-DNA complex in our EMSAs. A previous report showing that $P$. aeruginosa cells lacking PA5471 but possessing MexZ could not induce the expression of MexX by drug stimulation (Morita et al., 2006) also implied low probability of this direct interaction.

\section{Conclusion}

This PA5471-mediated transcriptional regulation mechanism seems to correspond to the production of similar cytotoxic signals in response to the administration of several different antibiotics, rather than corresponding to a signal specific to each, although PA5471 may play another initial role in maintaining cell homeostasis, and the drugefflux function of MexXY may be secondary to an unknown essential function. Elucidation of the transcriptional regulation of the PA5470-5471 operon might give us insight into the physiological function of the whole system.

Thus, we clarified the interaction between PA5471 and the MexZ-regulated expression of MexXY. However, the precise change in MexZ triggered by the interaction with PA5471 that leads to DNA-binding disability remains to be elucidated. The contribution of MexXY to aminoglycoside resistance in clinically isolated strains differs among strains, even when the mexX is highly expressed (Sobel et al., 2003). We also surveyed the expression levels of MexX and MexZ in clinically isolated aminoglycoside-resistant strains. The finding that almost all the aminoglycoside-resistant strains expressed MexX, but that expression of MexZ in these strains was diverse (data not shown) is consistent with the results of a previous report (Sobel et al., 2003). Functional assays and further examination of the expression profiles of PA5471 in these MexX-expressing AG-R strains are in progress in our laboratory.

\section{REFERENCES}

Aires, J. R., Kohler, T., Nikaido, H. \& Plesiat, P. (1999). Involvement of an active efflux system in the natural resistance of Pseudomonas aeruginosa to aminoglycosides. Antimicrob Agents Chemother 43, 2624-2628.

Aramaki, H., Sagara, Y., Kabata, H., Shimamoto, N. \& Horiuchi, T. (1995). Purification and characterization of a cam repressor (CamR) for the cytochrome P-450cam hydroxylase operon on the Pseudomonas putida CAM plasmid. J Bacteriol 177, 3120-3127.

Ausubel, F. M., Brent, R., Kingston, R. E., Moore, D. D., Seidman, J. G., Smith, J. A. \& Struhl, K. (1995). Current Protocols in Molecular Biology. Chichester: John Wiley.

Barcak, G. J., Chandler, M. S., Redfield, R. J. \& Tomb, J. F. (1991). Genetic systems in Haemophilus influenzae. Methods Enzymol 204, 321-342.

Bertrand, K. P., Postle, K., Wray, L. V., Jr \& Reznikoff, W. S. (1983). Overlapping divergent promoters control expression of Tn10 tetracycline resistance. Gene 23, 149-156. 
Daigle, D. M., Cao, L., Fraud, S., Wilke, M. S., Pacey, A., Klinoski, R., Strynadka, N. C., Dean, C. R. \& Poole, K. (2007). A protein modulator of multidrug efflux gene expression in Pseudomonas aeruginosa. J Bacteriol 189, 5441-5451.

Dean, C. R., Visalli, M. A., Projan, S. J., Sum, P. E. \& Bradford, P. A. (2003). Efflux-mediated resistance to tigecycline (GAR-936) in Pseudomonas aeruginosa PAO1. Antimicrob Agents Chemother 47, 972-978.

Eckert, B. \& Beck, C. F. (1989). Overproduction of transposon Tn10encoded tetracycline resistance protein results in cell death and loss of membrane potential. J Bacteriol 171, 3557-3559.

Forster, K., Helbl, V., Lederer, T., Urlinger, S., Wittenburg, N. \& Hillen, W. (1999). Tetracycline-inducible expression systems with reduced basal activity in mammalian cells. Nucleic Acids Res 27, 708-710.

Freundlieb, S., Baron, U., Bonin, A. L., Gossen, M. \& Bujard, H. (1997). Use of tetracycline-controlled gene expression systems to study mammalian cell cycle. Methods Enzymol 283, 159-173.

Gossen, M., Freundlieb, S., Bender, G., Muller, G., Hillen, W. \& Bujard, H. (1995). Transcriptional activation by tetracyclines in mammalian cells. Science 268, 1766-1769.

Grkovic, S., Brown, M. H., Schumacher, M. A., Brennan, R. G. \& Skurray, R. A. (2001). The staphylococcal QacR multidrug regulator binds a correctly spaced operator as a pair of dimers. J Bacteriol 183, 7102-7109.

Hagman, K. E., Pan, W., Spratt, B. G., Balthazar, J. T., Judd, R. C. \& Shafer, W. M. (1995). Resistance of Neisseria gonorrhoeae to antimicrobial hydrophobic agents is modulated by the mtrRCDE efflux system. Microbiology 141, 611-622.

Hillen, W. \& Berens, C. (1994). Mechanisms underlying expression of Tn 10 encoded tetracycline resistance. Annu Rev Microbiol 48, 345-369.

Hocquet, D., Nordmann, P., El Garch, F., Cabanne, L. \& Plesiat, P. (2006). Involvement of the MexXY-OprM efflux system in emergence of cefepime resistance in clinical strains of Pseudomonas aeruginosa. Antimicrob Agents Chemother 50, 1347-1351.

Jeannot, K., Sobel, M. L., El Garch, F., Poole, K. \& Plesiat, P. (2005). Induction of the MexXY efflux pump in Pseudomonas aeruginosa is dependent on drug-ribosome interaction. J Bacteriol 187, 5341-5346.

Klein, J. R., Henrich, B. \& Plapp, R. (1991). Molecular analysis and nucleotide sequence of the envCD operon of Escherichia coli. Mol Gen Genet 230, 230-240.

Llanes, C., Hocquet, D., Vogne, C., Benali-Baitich, D., Neuwirth, C. \& Plesiat, P. (2004). Clinical strains of Pseudomonas aeruginosa overproducing MexAB-OprM and MexXY efflux pumps simultaneously. Antimicrob Agents Chemother 48, 1797-1802.

Ma, D., Alberti, M., Lynch, C., Nikaido, H. \& Hearst, J. E. (1996). The local repressor AcrR plays a modulating role in the regulation of $a c r A B$ genes of Escherichia coli by global stress signals. Mol Microbiol 19, $101-112$

Masuda, N., Sakagawa, E., Ohya, S., Gotoh, N., Tsujimoto, H. \& Nishino, T. (2000a). Substrate specificities of MexAB-OprM, MexCDOprJ, and MexXY-OprM efflux pumps in Pseudomonas aeruginosa. Antimicrob Agents Chemother 44, 3322-3327.

Masuda, N., Sakagawa, E., Ohya, S., Gotoh, N., Tsujimoto, H. \& Nishino, T. (2000b). Contribution of the MexX-MexY-OprM efflux system to intrinsic resistance in Pseudomonas aeruginosa. Antimicrob Agents Chemother 44, 2242-2246.

Matsuo, Y., Eda, S., Gotoh, N., Yoshihara, E. \& Nakae, T. (2004). MexZ-mediated regulation of mexXY multidrug efflux pump expression in Pseudomonas aeruginosa by binding on the mexZmexX intergenic DNA. FEMS Microbiol Lett 238, 23-28.

McDermott, P. F., Walker, R. D. \& White, D. G. (2003). Antimicrobials: modes of action and mechanisms of resistance. Int J Toxicol 22, 135-143.
Mima, T., Sekiya, H., Mizushima, T., Kuroda, T. \& Tsuchiya, T. (2005). Gene cloning and properties of the RND-type multidrug efflux pumps MexPQ-OpmE and MexMN-OprM from Pseudomonas aeruginosa. Microbiol Immunol 49, 999-1002.

Morita, Y., Sobel, M. L. \& Poole, K. (2006). Antibiotic inducibility of the MexXY multidrug efflux system of Pseudomonas aeruginosa: involvement of the antibiotic-inducible PA5471 gene product. J Bacteriol 188, 1847-1855.

Okamoto, K., Gotoh, N. \& Nishino, T. (2002). Alterations of susceptibility of Pseudomonas aeruginosa by overproduction of multidrug efflux systems, MexAB-OprM, MexCD-OprJ, and MexXY/OprM to carbapenems: substrate specificities of the efflux systems. J Infect Chemother 8, 371-373.

Pearson, J. P., Pesci, E. C. \& Iglewski, B. H. (1997). Roles of Pseudomonas aeruginosa las and rhl quorum-sensing systems in control of elastase and rhamnolipid biosynthesis genes. J Bacteriol 179, 5756-5767.

Poole, K. (2001). Multidrug efflux pumps and antimicrobial resistance in Pseudomonas aeruginosa and related organisms. J Mol Microbiol Biotechnol 3, 255-264.

Poole, K. (2002a). Outer membranes and efflux: the path to multidrug resistance in Gram-negative bacteria. Curr Pharm Biotechnol 3, 77-98.

Poole, K. (2002b). Mechanisms of bacterial biocide and antibiotic resistance. J Appl Microbiol 92 (Suppl), 55S-64S.

Poole, K. (2005a). Efflux-mediated antimicrobial resistance. J Antimicrob Chemother 56, 20-51.

Poole, K. (2005b). Aminoglycoside resistance in Pseudomonas aeruginosa. Antimicrob Agents Chemother 49, 479-487.

Sanchez, P., Alonso, A. \& Martinez, J. L. (2002a). Cloning and characterization of SmeT, a repressor of the Stenotrophomonas maltophilia multidrug efflux pump SmeDEF. Antimicrob Agents Chemother 46, 3386-3393.

Sanchez, P., Rojo, F. \& Martinez, J. L. (2002b). Transcriptional regulation of mexR, the repressor of Pseudomonas aeruginosa mexABoprM multidrug efflux pump. FEMS Microbiol Lett 207, 63-68.

Schnappinger, D. \& Hillen, W. (1996). Tetracyclines: antibiotic action, uptake, and resistance mechanisms. Arch Microbiol 165, 359-369.

Smith, A. W. \& Iglewski, B. H. (1989). Transformation of Pseudomonas aeruginosa by electroporation. Nucleic Acids Res 17, 10509.

Sobel, M. L., McKay, G. A. \& Poole, K. (2003). Contribution of the MexXY multidrug transporter to aminoglycoside resistance in Pseudomonas aeruginosa clinical isolates. Antimicrob Agents Chemother 47, 3202-3207.

Teran, W., Krell, T., Ramos, J. L. \& Gallegos, M. T. (2006). Effectorrepressor interactions, binding of a single effector molecule to the operator-bound TtgR homodimer mediates derepression. J Biol Chem 281, 7102-7109.

Vogne, C., Aires, J. R., Bailly, C., Hocquet, D. \& Plesiat, P. (2004). Role of the multidrug efflux system MexXY in the emergence of moderate resistance to aminoglycosides among Pseudomonas aeruginosa isolates from patients with cystic fibrosis. Antimicrob Agents Chemother 48, 1676-1680.

Westbrock-Wadman, S., Sherman, D. R., Hickey, M. J., Coulter, S. N., Zhu, Y. Q., Warrener, P., Nguyen, L. Y., Shawar, R. M., Folger, K. R. \& Stover, C. K. (1999). Characterization of a Pseudomonas aeruginosa efflux pump contributing to aminoglycoside impermeability. Antimicrob Agents Chemother 43, 2975-2983.

Wright, G. D. (2003). Mechanisms of resistance to antibiotics. Curr Opin Chem Biol 7, 563-569.

Edited by: A. R. Walmsley 